This is an Open Access article distributed under the terms of the Creative Commons Attribution-Noncommercial License, which permits unrestricted use, distribution, and reproduction in any noncommercial medium, provided the original work is properly cited.

\title{
FILTER SIMULATIONS FOR THE SPICA MIRACLE INSTRUMENT IN COMBINATION WITH SAFARI
}

\author{
C. Pearson ${ }^{1,2,3}$, M. Negrello ${ }^{3}$, I. Perez-Fournon ${ }^{4}$, and K.G. Isaak ${ }^{5}$ \\ ${ }^{1}$ Space Science and Technology Department, Rutherford Appleton Laboratory, Chilton, Didcot, Oxfordshire OX11 0QX, UK \\ ${ }^{2}$ Department of Physics, University of Lethbridge, 4401 University Drive, Lethbridge, Alberta T1J 1B1, Canada \\ ${ }^{3}$ Department of Physics and Astronomy, Open University, Walton Hall, Milton Keynes MK7 6AA, UK \\ ${ }^{4}$ Instituto de Astrofsica de Canarias, Tenerife \\ ${ }^{5}$ Cardiff University, School of Physics Astronomy, Queens Buildings, The Parade, Cardiff, CF24 3AA, UK
}

\begin{abstract}
The Mid-Infra-Red Camera w/o LEns (MIRACLE) instrument is the proposed imaging camera for one of the the focal plane instruments on SPICA covering the wavelength range from 5-38 microns. Sub-micro-Jansky sensitivities are expected at wavelengths shortward of $10 \mathrm{mi}-$ crons, and sensitivities of the order of a few micro-Jy in the $10-40$ micron range. We present an initial investigation of the possible narrow filter combinations for MIRACLE, and the advantages of using MIRACLE and SAFARI in tandem to obtain photometric redshifts of high redshift sources. We apply our photometric redshift code that utilizes only the infrared spectra of the target sources and that can successfully and accurately constrain the redshift of our samples over the redshift $\mathrm{z} \sim 1-5$. We find that the addition of the SAFARI bands usually improves greatly the photo-z accuracy at redshifts $z>2$, extending out to redshifts of $\mathrm{z}=5$.
\end{abstract}

Key words: Galaxies: formation - Galaxies: evolution Missions: SPICA

\section{INTRODUCTION}

Galaxy surveys produce large statistical data sets for cosmological study. The first products from such surveys are usually the counts of the detected sources in a single detection waveband. Multi-band correlations provide additional information in the form of the colours of galaxies which can be used to segregate sources into general population classes and produce spectral energy distributions. However, a study of the exact astrophysics of the populations can only be made with the addition of distance information via measurement of the cosmological redshift. The acquisition of redshifts via the identification of spectral features provides a measurement of the redshift distribution (to constrain any evolution in the galaxy population), the calculation of luminosity functions, dust masses, star-formation rates and other astrophysical quantities. Unfortunately, spectroscopic redshifts often involve expensive,ground-based follow-up of faint infrared sources with complicated selection effects. The measurement of photometric redshifts (photo-z) via narrow band filters is therefore a popular intermediate alternative. Optical photo-z techniques have been well-used on large data sets, obtaining photometric accuracies of $\Delta z /(1+z)<1-2 \%$ (Wolf et al., 2004; Ilbert et al., 2008). Photometric redshifts for the Spitzer-SWIRE survey have been measured to an accuracy of $<5 \%$ for $10^{6}$ sources using a combination of optical and infrared filters (Rowan-Robinson et al., 2008) and most recently, Negrello et al. (2009) demonstrated a photometric accuracy of $<10 \%$ using solely midinfrared band data from the AKARI survey of the GOODS$\mathrm{N}$ field. Such mid-infrared photometric redshift techniques rely on the large number of PAH features found in the spectra of dusty galaxies (Puget \& Leger , 1989) and the $1.6 \mu \mathrm{m}$ bump, which arises due to photospheric emission from evolved stars (Sawicki, 2002) In this work we report on our initial studies of photometric redshift techniques for the Space Infrared Telescope for Cosmology \& Astrophysics (SPICA), investigating possible optimal narrowband filter combinations for the Mid-Infrared Camera w/o LEns (MIRACLE) instrument (Wada et al., 2009) with and without the supplementation of the SPICA FARinfra-red Instrument (SAFARI, Swinyard et al. (2009)) far-infrared wavebands. MIRACLE is an imaging camera \& low-resolution spectrometer consisting of 2 cameras with filter wheels covering the wavelength range from 5$27 \& 20-38 \mu \mathrm{m}$ respectively, with separate fields of view of $6^{\prime} \times 6^{\prime}$. The projected sensitivities are sub- $\mu \mathrm{Jy}$ at wavelengths $<10 \mu \mathrm{m}$, and of the order of a few $\mu \mathrm{Jy}$ at wavelengths $10-40 \mu \mathrm{m}$. For this study we also assume SAFARI $5 \sigma$ sensitivities (over a $2^{\prime} \times 2^{\prime} \mathrm{FoV}$ ) of 11,13 and $18 \mu \mathrm{Jy}$ in the $35-60,60-110 \& 110-210 \mu \mathrm{m}$ bands respectively.

\section{Simulations}

For our investigation of MIRACLE filter combinations we assume MIRACLE filters with a constant $\lg \lambda / \Delta \lambda=1$ (i.e. a resolution of $\Delta \lambda / \lambda=0.1$ ). A maximum of 12 filters are considered at $5,8,11,14,17,20 \mu \mathrm{m}$ and $23,26,29,32,35$, $38 \mu \mathrm{m}$ for the two cameras respectively. We then investigate the following cases of;

- 6 MIRACLE filter combination $(8,14,20,26,32,38 \mu \mathrm{m})$

- 8 MIRACLE filter combination $(5,8,11,14,20,26$, $32,38 \mu \mathrm{m})$

- 12 MIRACLE filter combination $(5,8,11,14,17,20$, $23,26,29,32,35,38 \mu \mathrm{m})$ 


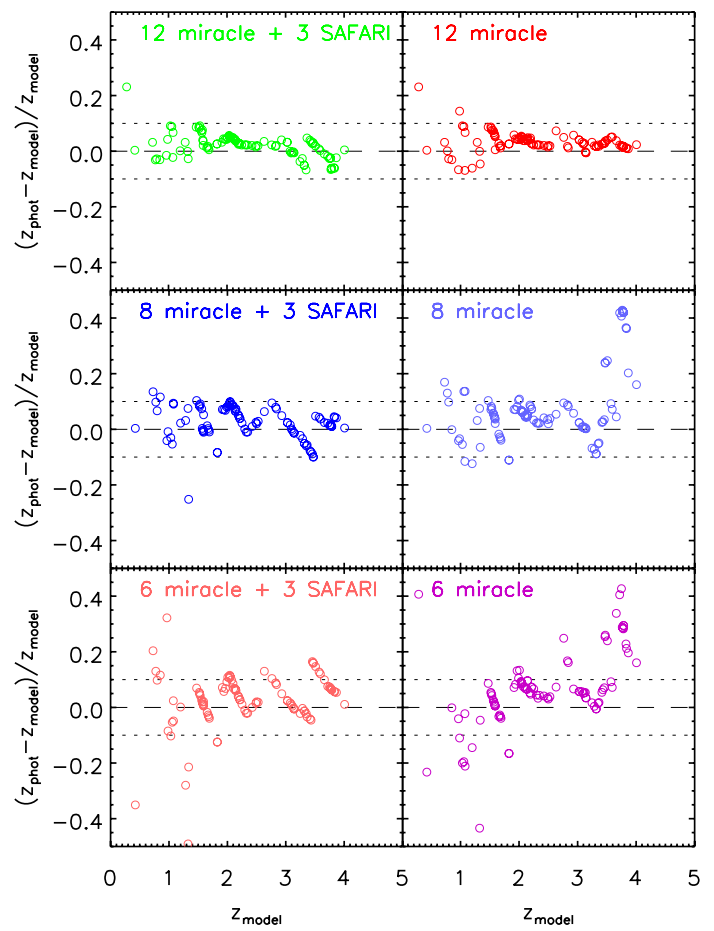

Figure 1. Photo-z results for individual galaxy populations: starburst galaxies. The difference in the estimated photometric and model redshift from the catalogue (divided by $(1+$ $\left.z_{\text {modelredshift }}\right)$ ) is plotted against the model redshift from the catalogue. A positive difference implies an over-estimation of the redshift and a negative difference correspondingly implies an under-estimation of the redshift.

- MIRACLE configurations with and without the addition of SAFARI bands

To test the effectiveness of our photo-z algorithm we create a synthetic catalogue of sources based on the galaxy evolution models of Pearson et al. (2007) which have been shown to provide excellent fits to galaxy counts from 1$1400 \mu \mathrm{m}$ (Pearson \& Khan, 2009). The evolutionary model is segregated into 5 broad population categories, a local non-evolving quiescent normal galaxy population, AGN dust torii, and 3 evolving starforming populations defined in increasing luminosity of starbursts $\left(\mathrm{L}_{\mathrm{IR}}<10^{11} \mathrm{~L}_{\odot}\right)$, luminous (LIRGs $10^{11} \mathrm{~L}_{\odot}<\mathrm{L}_{\mathrm{IR}}<10^{12} \mathrm{~L}_{\odot}$ ) and ultraluminous (ULIRGs $\mathrm{L}_{\mathrm{IR}}>10^{12} \mathrm{~L}_{\odot}$ ) infrared galaxies. A total of 11 template spectra (2 normal, 2 starburst, 3 LIRG, 2 ULIRG \& 2 AGN) are drawn from the spectral libraries of Efstathiou \& Rowan-Robinson (1995), Efstathiou et al. (2000), Efstathiou \& Rowan-Robinson (2003). Note that the ULIRG templates consist of one colder template and one hot template, based on the archetypal ULIRGs Arp220 and Mk231 respectively. The spectral templates are smoothed by the MIRACLE filter bandwidths and a random variation of $15 \%$ in the flux introduced to provide a wide and varied range of spectra from a relatively small number of input templates. A synthetic catalogue is then created covering 0.1 square degrees to a sensitiv-

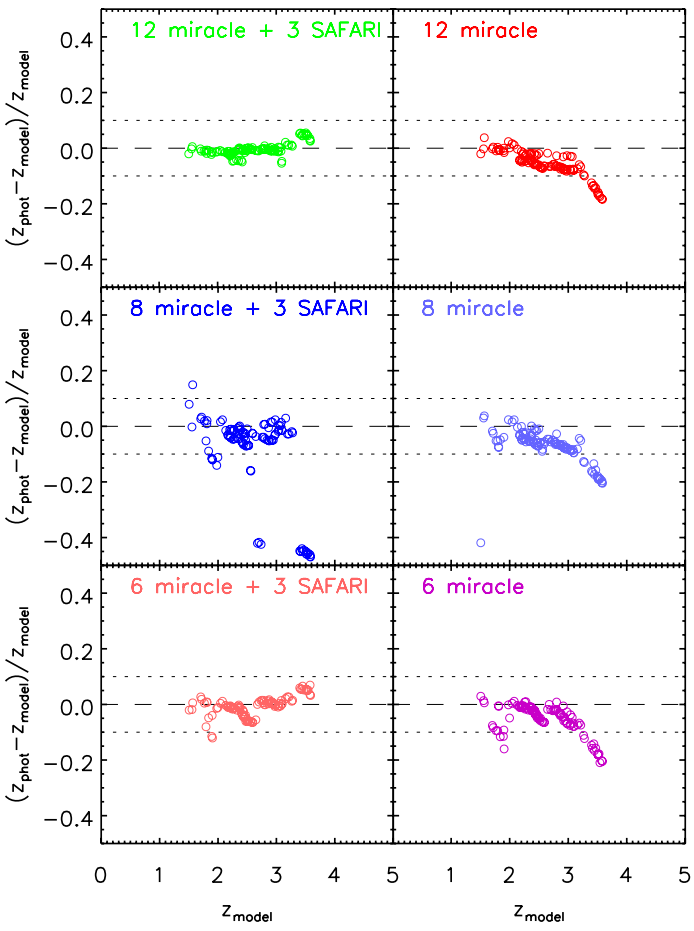

Figure 2. Photo- $z$ results for individual galaxy populations: luminous infrared galaxies (LIRG). Axes are the same as Figure 1 .

ity of $1 \mu \mathrm{Jy}$ at $38 \mu \mathrm{m}$, with source redshifts and fluxes calculated in all MIRACLE and SAFARI bands.

The simulations are then analysed using the photometric redshift code of Negrello et al. (2009). This photo-z algorithim uses the spectral starburst template libraries of Takagi et al. (2003), Takagi et al. (2004). The library templates are convolved with the response functions of the instruments and the best fit parameters of library models to the synthetic catalogue sources, including the redshift, are obtained by minimizing the $\chi^{2}$ distribution. In this work we concentrate on the analysis of the star-forming galaxies and address, in particular, the characteristics of the AGN in future work.

\section{Results}

In Figures 1,2, 3 \& 4 the results for the individual, evolving star-forming populations are shown (starburst, LIRG, cold-ULIRG \& hot-ULIRG respectively). The difference in the estimated photometric and model redshift from the catalogue divided by $(1+$ model redshift $)$, i.e., $\left(z_{\text {photo }}-\right.$ $\left.z_{\text {model }}\right) /\left(1+z_{\text {model }}\right)$ is plotted against the model redshift from the catalogue $\left(z_{\text {model }}\right)$. A positive difference implies an over-estimation of the redshift by the photo-z technique and a negative difference correspondingly implies an under-estimation of the redshift. Results are plotted for the cases of 6,8 and 12 MIRACLE bands, both with and without the addition of the SAFARI bands. Figure 1 plots the results for the starburst population. The number 


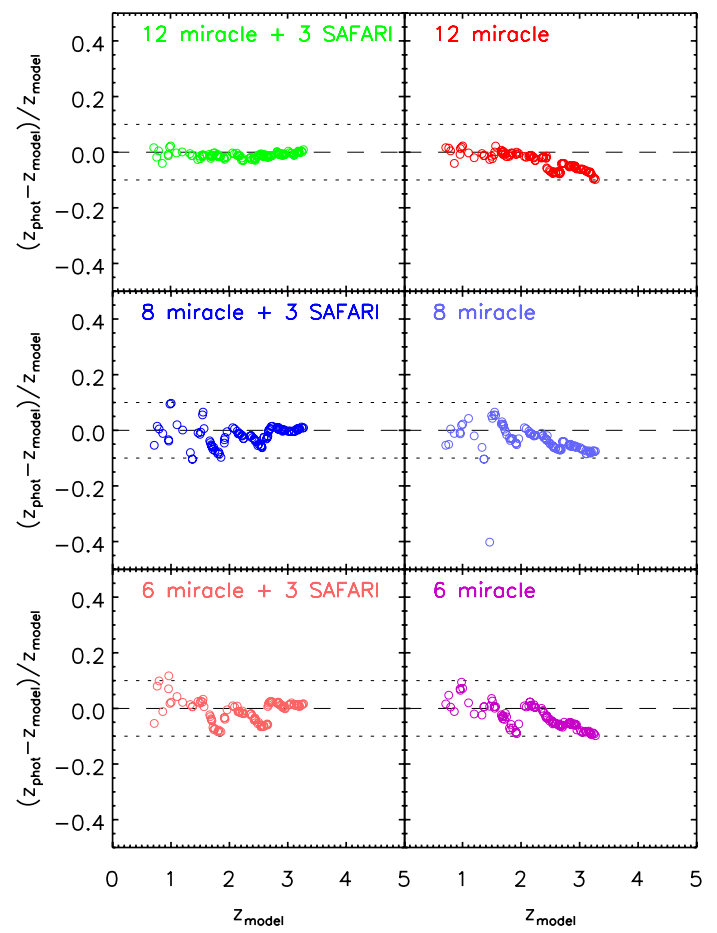

Figure 3. Photo- $z$ results for individual galaxy populations: cool (Arp220-like) ultraluminous infrared galaxies (ULIRG). Axes are same as Figure 1.

of filters does not significantly affect the photo-z accuracy at $\mathrm{z}<2-3$. At higher redshifts, for the case of 12 MIRACLE filters, the $1.6 \mu \mathrm{m}$ bump is well sampled from $3<\mathrm{z}<4$ and provides an accurate redshift estimate even without the support of SAFARI. However, with only 6 or 8 MIRACLE bands the $1.6 \mu \mathrm{m}$ bump is not well sampled and the support of the SAFARI filters become crucial to provide reasonably good photo-z estimates to $z=4$ by sampling the redshifted PAH and silicate features. Figure 2 plots the results for the LIRG population. Note that the catalogue LIRG templates have prominent $6.2+7.7 \mu \mathrm{m}$ PAH emission and $9.7 \mu \mathrm{m}$ silicate absorption features, and at $\mathrm{z}>3$ the $9.7 \mu \mathrm{m}$ silicate feature is redshifted into the shortestwavelength SAFARI filter aiding the photo-z accuracy at these redshifts. For the case of 8 MIRACLE +3 SAFARI filters, the photo- $z$ estimates at $\mathrm{z}>3$ are actually worse than the 6 filter case. This is due to a systematic difference between the model templates and the templates used for the photo-z estimation. Passing from 8 to 6 MIRACLE filters actually under-samples the $1.6 \mu \mathrm{m}$ bump, and the photo-z improves since there are fewer features to simultaneously account for. For the 12 MIRACLE filters + SAFARI bands case, the photo-z accuracy is excellent because all features are better sampled, and there is less degeneracy in fitting the features. Figure 3 plots the results for the cold-ULIRG population (modelled on the archetypal ULIRG Arp220). Here, the photo-z accuracy achieved is reasonably good for any combination of

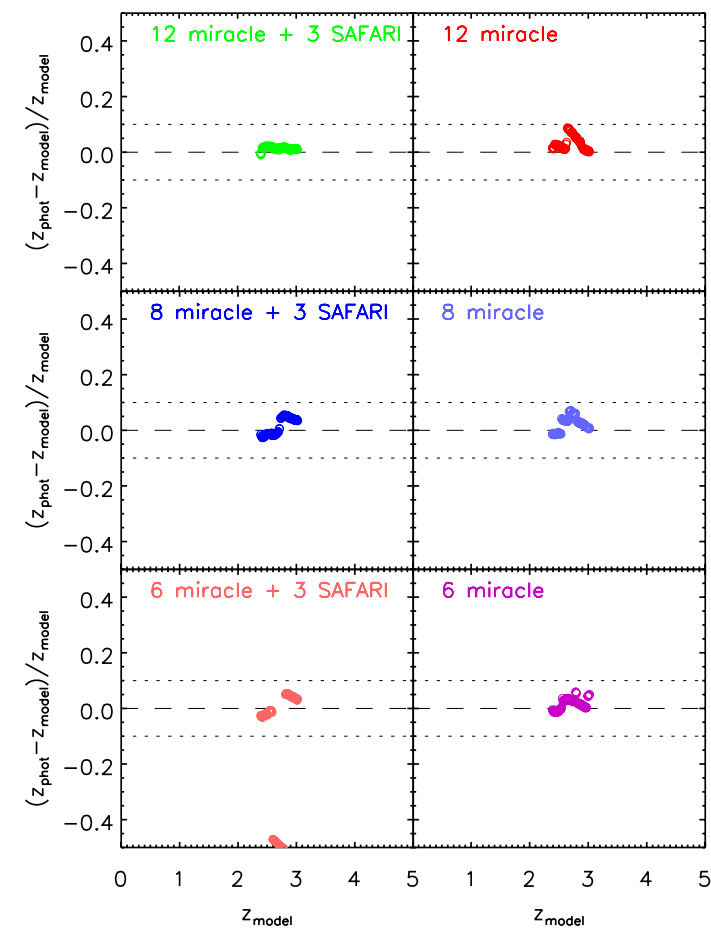

Figure 4. Photo- $z$ results for individual galaxy populations: hot (Mk231-like) ultraluminous infrared galaxies (ULIRG). Axes are same as Figure 1.

filters, however when the 3 SAFARI filters are removed from the analysis the derived photo- $z$ tends to be underestimated at $\mathrm{z}>3$ since the silicate absorption feature is shifted into the $45 \mu \mathrm{m}$ SAFARI filter (using the MIRACLE filters alone omit this important redshift indicator). Figure 4 plots the results for the hot-ULIRG population (modelled on the archetypal ULIRG mk231). Although these were rare objects in the simulation, the photo-z accuracy achieved over the probed redshift range $2<\mathrm{z}<3$ was good for any choice of the number of the filters. One exception is the case of 6 MIRACLE +3 SAFARI filters in the narrow redshift window $2.6<\mathrm{z}<2.8$, not seen when 6 MIRACLE filters alone are used. In this redshift range the $9.7 \mu \mathrm{m}$ silicate absorption is mistaken for a redshifted $18 \mu \mathrm{m}$ silicate feature when the SAFARI filters are added and this degeneracy destroys the photo-z fitting. Note that although the $1.6 \mu \mathrm{m}$ bump is visible in the spectra of this population, it is not sampled in the case of 6 MIRACLE filters. For the 8 or 12 MIRACLE filter case the feature is identified and thus the accuracy is improved.

Finally, in Figure 5 the combined results from the previous figures are collated together. It can be seen that assuming 6 or 8 bands for MIRACLE gives accurate photo-z estimates out to redshifts of $\sim 3$. Increasing the number of MIRACLE bands to 12 extends this accuracy to out to redshifts of $\sim 4$. Utilizing 12 MIRACLE bands also reduces the degeneracy due to aliasing in the photo-z fitting at moderate redshifts, $1<\mathrm{z}<2$. The advantage of SAFARI 
is at high redshift, where utilizing the SAFARI bands usually greatly improves the photo-z accuracy at redshifts $\mathrm{z}>$ 2 , extending out to redshifts of $\mathrm{z} \sim 4$ as the prominent midinfrared features are redshifted into the SAFARI bands. In this initial analysis we have not explicitly included the effect of the AGN on our photometric redshift estimates. In general, the featureless spectra of AGN make photo-z impractical and the photometric redshift technique fails to produce accurate redshifts. However, the dispersion in photo-z for the non-AGN populations is so small that all outliers are in fact AGN, especially if the SAFARI bands are included in the analysis

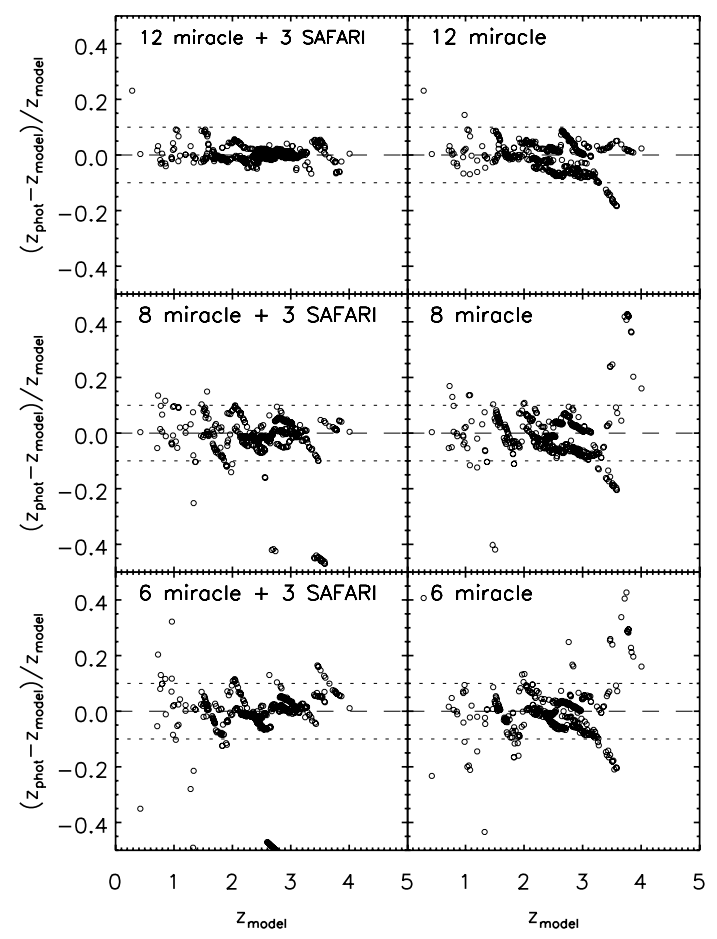

Figure 5. Photo- $z$ results for all galaxy populations from Figures 1,2, 3 \& 4 . Axes are same as Figure 1.

\section{Summary}

We have carried out an initial investigation into the possible narrow filter combinations for the MIRACLE instrument on SPICA and the advantages of using the MIRACLE and SAFARI instruments in tandem to obtain photometric redshifts of high redshift sources. We analysed the results for the cases of 6,8 and 12 MIRACLE bands both with, and without, the addition of the SAFARI bands. Obviously more bands result in better photometric accuracy, but the configuration of 12 MIRACLE bands results in zero outliers for the photo-z (excluding AGN) with accuracies of a few \%. This study concludes that mid-infrared filters spaced every $3-4 \mu \mathrm{m}$ in the SPICA spectral range can provide high-accuracy photometric redshifts and MIR-
FIR spectral classification. It is noted that although AGN are poorly modelled by photo-z techniques in general, they are in fact the only outliers in most cases.

We conclude that large numbers of objects over wide fields can be observed in a reasonable amount of time to deep fluxes, allowing cosmological studies without the immediate need of follow-up spectroscopy in other spectral ranges. As an example, considering the Spitzer MIPS$24 \mu \mathrm{m}$ sources in the GOODS-N field: a survey to the depth of $\mathrm{S}_{24}=80 \mu \mathrm{Jy}$ would detect $\sim 1200$ sources over a in $10^{\prime} \times 15^{\prime}$ area, corresponding to 8 sources / $\operatorname{arcmin}^{2}$. Thus, we could expect $8 \times 6^{\prime} \times 6^{\prime}=288$ sources per MIRACLE FOV and $8 \times 2^{\prime} \times 2^{\prime}=32$ sources per SAFARI FOV to $\mathrm{S}_{24}=80 \mu \mathrm{Jy}$. We could therefore envisage a SPICA multi-band survey of 2 square degrees (e.g. the COSMOS (Scoville et al. , 2007) field $=48$ GOODS fields) to a midinfrared depth of $80 \mu \mathrm{Jy}$ detecting $\sim 58,000$ sources, the majority of which it would be plausible to obtain photometric redshifts for. Such large statistical samples, with critical redshift information on dusty starburst galaxies, would provide extremely valuable constraints on evolutionary scenarios and hierarchal formation models

\section{ACKNOWLEDGEMENTS}

The authors thank the conference organizers for providing an excellent forum for discussions on the SPICA project.

\section{REFERENCES}

Efstathiou, A., Rowan-Robinson, M., 1995, MNRAS, 273, 649

Efstathiou, A., Rowan-Robinson, M., Siebenmorgen, R., 2000, MNRAS, 313, 734

Efstathiou, A., Rowan-Robinson, M., 2003, MNRAS, 343, 322

Ilbert, O., Salvato, M., Capak, P. et al., 2008, ASP Conference Series, 399, 169

Negrello, M., Serjeant, S., Pearson, C. et al. 2009, MNRAS, 394, 375

Pearson, C., Jeong W-S., Matsuura, S. et al. 2007, Adv.Space Res. 40, 605

Pearson, C., Khan S.A. 2009, MNRAS, in press, astroph/0906.4120

Puget, J.L., Leger, A., 1989, ARAA, 27, 161

Rowan-Robinson, M., Babbage T., Oliver S. et al. 2008, MNRAS, 386, 697

Sawicki, M., 2002, AJ, 124, 3050

Scoville, N., Aussel, H., Brusa, M., 2007, ApJS, 172, 1

Swinyard B. et al. 2009, these proceedings

Takagi, T., Arimoto, N., Hanami, H., 2003, MNRAS, 340, 813

Takagi, T., Hanami, H., Arimoto, N., 2004, MNRAS, 355, 424

Wada, T. et al. 2009, these proceedings

Wolf C., Meisenheimer, K., Kleinheinrich, M.A. et al. 2004, A\&A, 421, 913 